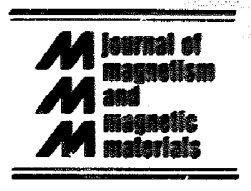

\title{
Measuring dc susceptibility of particulate media
}

\author{
J.S. Yang ${ }^{\text {a, }}$, J. Lee ${ }^{b}$, I. Klik ${ }^{c}$, C.R. Chang ${ }^{c}$ \\ a Division of General Education, National Taiwan Ocean Universiry, Keelong, Taiwan \\ ${ }^{b}$ Department of Electrical Engineering, Chang Gung College of Medicine and Technology, Kwei-San, Tao-Ywan, Taiwan \\ 'Department of Physics, National Taiwan University, Taipei, Taiwan
}

\section{Alstract}

The initial dc susceptioility $\chi$ of an array of single domain uniaxial particles is calculated as a function of temperature, sample texture, distribution of activation volumes and measurement duration. Interactions are modeled by the mean field approximation within which a self-consistent expression for $\boldsymbol{X}$ is derived.

The response of a thermally relaxing system to outside stimuli is delayed since thermal relaxation takes place on a finite time scale. We consider here a uniaxial, single domain ferromagnetic particle with (activation) volume $V$, anisotropy constant $K$ and saturation magnetization $M_{\mathrm{s}}$, an external field is applied in the $z$-direction with which the particle's easy axis spans an angle $\beta$. The energy of the system is then $E / K V=\sin ^{2}(\theta-\beta)-2 h \cos \theta$, where $h=H / H_{\mathrm{n}}, H_{\mathrm{n}}=2 K / M_{\mathrm{s}}$, is the reduced applied field and $\theta$ is the angle spanned by the magnetization vector $M$ and the $z$-axis. At sufficiently small fields the energy function has two minima at $\theta_{1}$ and $\theta_{2}$. We shall treat an array of identical noninteracting particles as having two levels [1] (low temperature assumption, $q \gg 1$, see below) so that its reduced magnetization $m=M_{z} / M_{\mathrm{s}}=$ $n_{1} \cos \theta_{1}+n_{2} \cos \theta_{2}$ where $n_{i}$ is the probability that the $i$-th local minimum is occupied. The probabilities $n_{i}$ satisfy the obvious relation $n_{1}+n_{2} \equiv 1$ and the master equation [1]

$\dot{n}_{1}=-\kappa_{12} n_{1}+\kappa_{21} n_{2}=-\dot{n}_{2}$,

$\dot{n}_{i}=\mathrm{d} n_{i} / \mathrm{d} t$, where $\kappa_{i j}(h)=\kappa_{i \rightarrow j}=f_{0} \exp \left[-Q_{i}(h) /\right.$ $\left.k_{\mathrm{B}} T\right]$ is the transition rate of the process $i \rightarrow j$, the prefactor $f_{0} \approx \mathrm{e}^{25} \mathrm{~Hz}$ [1-3], $Q_{i}$ is the barrier height to be overcome on leaving the $i$-th well and $T$ is temperature.

Let us now assume that the system initially was in a demagnetized state in zero field with $n_{1}=n_{2}=\frac{1}{2}$ (thermal equilibrium) and that an infinitesimal field $h>0$ is applied in the $z$-direction at time $t=0$ and maintained. Substituting the expansion $\theta_{i} \rightarrow \theta_{i}+h \delta \theta_{i}$ into the extremal equation $\mathrm{d} E / \mathrm{d} \theta=0$ we find the perturbed positions of the barrier and local minima. The expansions $n_{i} \rightarrow n_{i}+h \delta n_{i}$

\footnotetext{
* Corresponding author. Fax: +886-2-46200724.
}

and $\kappa_{i j}(h) \approx \kappa_{i j}(0)+h \kappa_{i j}^{\prime}(0)$ in Eq. (1) yield, in the first order of $h$, a differential equation for $\delta n_{i}$ with the initial condition $\delta n_{i}(0)=0$. The perturbed reduced magnetization becomes $m \rightarrow m+h \delta m$ and, by definition, $\delta m$ is the reduced initial dc susceptibility $\chi_{\beta}(T, t)$ which finally takes on the form

$\chi_{\beta}(T, t)=\sin ^{2} \beta+2 q\left(1-\mathrm{e}^{-2 \kappa t}\right) \cos ^{2} \beta$,

where $q=K V / k_{\mathrm{B}} T$ is a reduced barrier height and $\mathrm{x}=$ $f_{0} \mathrm{e}^{-q}$ is the relaxation rate in zero field. The time independent term corresponds to a shift in the positions of the local minima and the remairing term describes the gradual relaxation towards the new equilibrium state at field $\boldsymbol{h}$. If $\kappa t \gg 1$, Eq. (2) yields the usval equilibrium susceptibility whereas at $\kappa t \ll 1$ the system cannot relax daring the measurement period and Fig. 1 shows the function $\left.\chi_{\beta}(T)\right|_{1=\text { coast }}$ with a sharp peak at the blocking temperature [2] where $\kappa(T) t \approx 1$. However, when averaged [3] over $V$ Eq. (2) yields a very broad peak for which the concept of blocking temperature is no longer well defined. The activation volume is usually [2] assumed to bave a log-normal distribution $P(V) \sim V^{-1} \exp \left[-\ln ^{2}\left(V / V_{0}\right) /\right.$ $\left(2 \rho^{2}\right)$, the parameter $\rho$ expresses the distribution width. The susceptibility of a textured [4] array is obtained by averaging Eq. (2) with respect to the inclination angle $\beta$.

Interparticle interactions may be included within the mean field approximation [5] in which the applied field $h$ is replaced by the effective field $h_{\text {eff }}=\boldsymbol{h}+\boldsymbol{\alpha}\langle\boldsymbol{m}\rangle$ where $\langle m\rangle$ is the instantaneous net magnetization of the array and $\alpha$ is a small interaction constant. For simplicity we shall assume that the texture of the array has axial symmetry so that the vector $\langle m\rangle$ is in the $z$-direction. Eq. (1) yields

$\delta \dot{n}_{1 \beta}=-2 \kappa \delta n_{1 \beta}+2 q \kappa(1+\langle\delta m\rangle) \cos \beta$, 


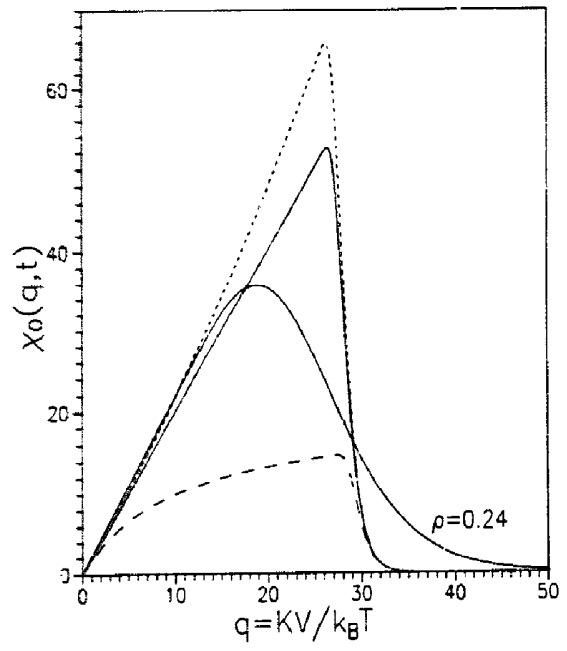

Fig. 1. The reduced susceptibility (2) plotted versus the reduced iaverse temperature $q=K V / k_{\mathrm{B}} T$ (solid unlabeled line). Also shown is the mean field susceptibility (6) with $\alpha=4 \times 10^{-3}$ (short dash) and $a=-5 \times 10^{-2}$ (long dash) and the susceptibility (2) averaged over activation volumes with log-normal distribution and $\rho=0.24$ (solid labeled line). For this curve $q=$ $K V_{0} / k_{B} T$. In all cases the inclination angle $\beta=0$ and the wating time $t=10 \mathrm{~s}$. At larger waiting times the peaks shift towards lower temperatures.

for every $\beta$ and the unknown function $\langle\delta m(t)\rangle$ satisfies the relation

$\bar{s}+2\left\langle\delta n_{1 \beta} \cos \beta\right\rangle=\langle\delta m\rangle(1-\alpha \bar{s})$,

obtained by perturbing the mean magnetization $\langle m\rangle, \bar{s}=$ $\left\langle\sin ^{2} \beta\right\rangle$ and $\bar{c}=\left\langle\cos ^{2} \beta\right\rangle$ for brevity. Substituting the solution of Eq. (3), with $\delta_{1 \beta}(0)=0$, into Eq. (4) results in a Voltera integral equation

$$
\begin{aligned}
\bar{s} & \left.+4 q \kappa \bar{c} \int_{0}^{t} \mathrm{~d} t^{\prime} \mathrm{e}^{-2 \kappa\left(t-t^{\prime}\right.}\right)\left[1+\alpha\left\langle\delta m\left(t^{\prime}\right)\right\rangle\right] \\
& =\langle\delta m(t)\rangle(1-\alpha \bar{s}),
\end{aligned}
$$

whose solution $\chi(T, t)=\langle\delta m(t)\rangle$ is

$\chi(T, t)=\sigma^{-1} \bar{s} \mathrm{e}^{-2 \Gamma t}+\xi(1-\alpha \xi)^{-1}\left(1-\mathrm{e}^{2 \Gamma t}\right)$,

$\sigma=1-\alpha \bar{s}, \xi=\bar{s}+2 q \bar{c}$ and the new relaxation rate $\Gamma=$ $\kappa \sigma^{-1}(1-\alpha \xi)$. Interactions alter the minima positions, the relaxation rate and the asymptotic $(t \rightarrow \infty)$ susceptibility. Ferromagnetic coupling, $\alpha>0$, slows down the relaxation rate and increases the slope of the initial dc susceptibility while antiferromagnetic coupling has the opposite effect (see Fig. 1). Textured arrays yield a variety of similar curves.

So far the perturbing field was turned on instanteneously, a more detailed analysis follows: Let $h(t)=h t / t_{1}$ $\therefore$ the time interval $0 \leq t \leq t_{1}$. Eq. (1) has, in this case, the solution

$\delta n_{1 \beta}\left(t_{1}\right)=q\left[1-f\left(t_{1}\right)\right] \cos \beta$

with $f(t)=\left(1-\mathrm{e}^{-2 \kappa t}\right) /(2 \kappa t)$. At time $t_{1}$ the field reaches its final value at which it is maintained for time $t_{2}$. The riet response of the noninteracting array is then

$\chi_{\beta}\left(T, t_{\mathrm{i}}+t_{2}\right)=\sin ^{2} \beta \div 2 q\left[1-\mathrm{e}^{-2 \kappa t_{2}} f\left(t_{1}\right)\right] \cos ^{2} \beta$.

The formulae (2), (6) and (7) show that the initial susceptibility of a particulate system depends on the distribution of activation volumes, on the interaction strength, on the texture of the system and on measurement duration.

\section{References}

[1] J.J. Lu, H.L. Huang, C.R. Chang and I. Klik, J. Appl. Phys. 75 (1994) 5499.

[2] I. Klik, C.R. Chang and H.L. Huang, Phys. Rev. B 47 (1993) 8605.

[3] I. Klik and C.R. Chang, Phys. Rev. B 47 (1993) 909l.

[4] A. Hoare, R.W. Charticti, W. Scimitit aud A. Eiling, I. Phys. D: Appl. Phys. 26 (1993) 461.

[5] D.L. Atherton, IEEE Trans. Magn. 26 (1990) 3059. 